Supplement of The Cryosphere, 9, 2119-2134, 2015

http://www.the-cryosphere.net/9/2119/2015/

doi:10.5194/tc-9-2119-2015-supplement

(C) Author(s) 2015. CC Attribution 3.0 License.

(c) (i)

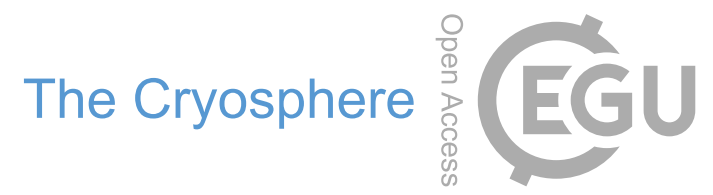

Supplement of

\title{
A prognostic model of the sea-ice floe size and thickness distribution
}

\section{Horvat and E. Tziperman}

Correspondence to: C. Horvat (horvat@ fas.harvard.edu)

The copyright of individual parts of the supplement might differ from the CC-BY 3.0 licence. 


\title{
A prognostic model of the sea ice floe size and thickness distribution
}

\author{
Christopher Horvat and Eli Tziperman
}

School of Engineering and Applied Sciences and Department of Earth and Planetary Sciences, Harvard University

\section{Supplementary Materials}

\section{Contents}

S1 Sensitivity tests 2

S1.1 Thermodynamics . . . . . . . . . . . . . . 2

S1.2 Mechanics . . . . . . . . . . . . . . . . . 4

S1.3 Wave-induced fracture . . . . . . . . . . . . . 5

S1.4 Attenuation model . . . . . . . . . . . . . . . 7

S2 Numerical convergence tests 8

S2.1 Mechanics run . . . . . . . . . . . . . . . . . 10

S2.2 Wave-induced fracture run . . . . . . . . . . . . . . . 11

S3 Calculation of Fractured Floe Size Distribution 11 


\section{S1 Sensitivity tests}

We examine the sensitivity of model results to perturbations in the set of parameters listed, along with sensitivity ranges, in Table S1, The perturbed parameters represent each of the model components. These tests demonstrate the robustness of the model to changes in the main model parameters, at the same time indicating the need to further constrain the relevant parameters.

\section{S1.1 Thermodynamics}

Model parameters that govern the thermodynamic model component include the pancake floe size $r_{\text {min }}$, pancake floe thickness $h_{\text {min }}$, and width of the lead region, $r_{\mathrm{lw}}$. To examine the model sensitivity to changes in these parameter values, the model is initialized with zero ice concentration, with only the thermodynamic component of the model enabled. The external forcing is a net cooling heat flux $Q_{e x}=-50 \mathrm{~W} / \mathrm{m}^{2}$, of which a proportion equal to $(1-c) Q_{e x}$, where $c$ is the ice concentration, is applied to water (assumed to be at its freezing temperature). This cooling over water is further decomposed into an "open water" cooling of magnitude $Q_{o}$, which leads to the growth of ice pancakes, and a "lead" cooling of magnitude $Q_{l}$ that leads to lateral and basal freezing, as outlined in the manuscript (Sec. 2.1). The net cooling in the region covered by ice has magnitude $c Q_{e x}$, and leads to only lateral and basal freezing, not to the formation of ice pancakes. To maintain a fixed grid in size and thickness space across all experiments, the pancake floe size

\begin{tabular}{|c|l|l|c|}
\hline Parameter & Description & component & Range \\
\hline$r_{\min }$ & Pancake floe size & thermo & $1 \mathrm{~m} \pm 0.5 \mathrm{~m}$ \\
$r_{\text {lw }}$ & Lead region width & thermo & $1 \mathrm{~m} \pm 0.5 \mathrm{~m}$ \\
$h_{\text {min }}$ & Pancake floe thickness & thermo & $0.2 \mathrm{~m} \pm 0.1 \mathrm{~m}$ \\
$\delta_{\text {ridge }}$ & Ridge width & mechanics & $5 \mathrm{~m} \pm 2.5 \mathrm{~m}$ \\
$k_{\text {ridge }}$ & Ridging thickness mult. & mechanics & $5 \pm 2$ \\
$v_{\mathrm{g}}$ & Wave-ice group velocity & waves & $\left(1 \pm \frac{1}{2}\right) \cdot \frac{1}{2} \sqrt{\mathrm{g} / \mathrm{k}} \mathrm{m} / \mathrm{s}$ \\
$\epsilon_{\text {crit }}$ & Crit. failure threshold & waves & $5 \cdot 10^{-6}-5 \cdot 10^{-5} 1 / \mathrm{s}$ \\
$\alpha$ & Attenuation Coefficient & waves & KM08 or BS12 \\
\hline
\end{tabular}

Table S1: Parameters varied in sensitivity tests, with the range of values used in sensitivity tests 
and thickness are specified separately from the smallest resolved floe size and thickness, which is held constant in these runs, equal to $0.5 \mathrm{~m}$ and $0.1 \mathrm{~m}$, respectively. Each run has an evenly spaced grid of 400 floe sizes from $0.5 \mathrm{~m}$ to $200 \mathrm{~m}$, and 14 floe thicknesses from $0.1 \mathrm{~m}$ to $2.7 \mathrm{~m}$.

First, the pancake ice thickness, $h_{\mathrm{min}}$, is varied from $0.1 \mathrm{~m}$ to $0.3 \mathrm{~m}$. Fig. S1 shows the model response in terms of the total ice volume (Fig. S1a), ice concentration (Fig. S1b) and open water cooling $Q_{o}$ (Fig. S1c). All runs have the same ice volume over time, since the net cooling is fixed. When the specified pancake thickness is larger, the added area of pancakes formed by the same net cooling must decrease, as volume is conserved. Accordingly, after 15 days the ice concentration in the experiment where $h_{\min }=.3 \mathrm{~m}$ is $45 \%$ of that in the experiment where $h_{\min }=.1 \mathrm{~m}$.
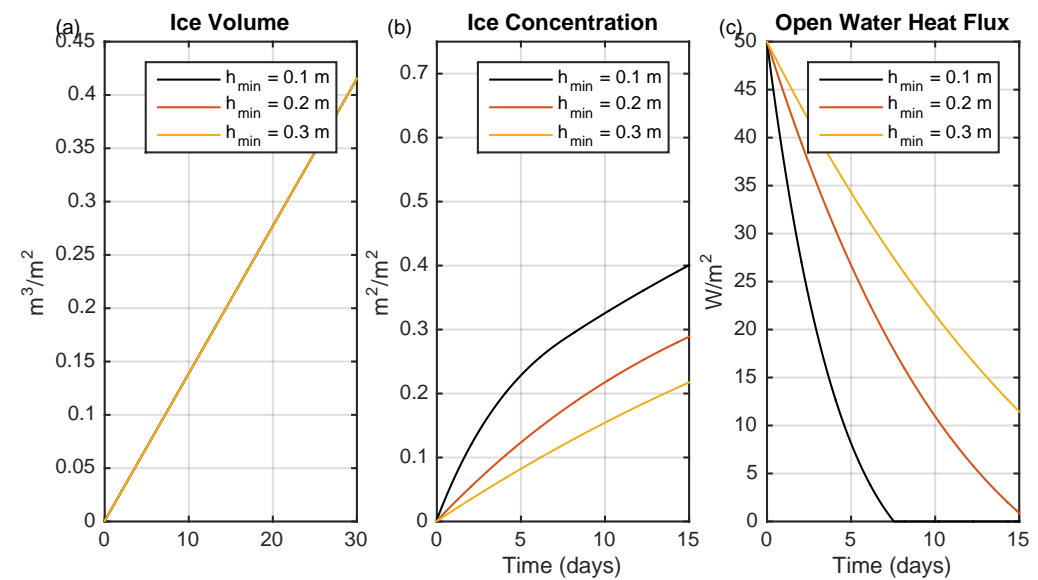

Figure S1: Sensitivity to the variation of the pancake ice thickness $h_{\min }$ from $0.1 \mathrm{~m}$ to $0.3 \mathrm{~m}$, for an initially uncovered sea surface with a net applied cooling of $50 \mathrm{~W} / \mathrm{m}^{2}$. (a) Ice volume, (b) ice concentration, and (c) net heat flux to the open water region for each run. Black lines correspond to default model values, $h_{\min }=0.1 \mathrm{~m}$.

We next separately vary the lateral size of ice pancakes, $r_{\min }$, and the width of the lead region, $r_{\mathrm{lw}}$, from $0.5 \mathrm{~m}$ to $1.5 \mathrm{~m}$ (Fig. S2). The ice volume is the same across both sets of runs (Fig. S2a,d), since the net cooling is fixed. First, we perturb $r_{\min }$ (Fig. S2 a-c). Since a portion of the cooling in the lead region contributes to the vertical growth of existing floes, cooling in the open water region leads to higher rates of ice concentration growth. 
Thus, decreasing the size of the lead region leads to an increase in the rate of increase of ice concentration. Increasing the pancake floe size leads to an increase in the net open water heat flux, and therefore a more rapid increase in the ice concentration (Fig. S2b,c). After 15 days, ice concentration increases by $35 \%$ between the run in which $r_{\min }=0.5 \mathrm{~m}$ and the run in which $r_{\min }=1.5 \mathrm{~m}$. In the second set of experiments, $r_{\mathrm{lw}}$ varies from $0.5 \mathrm{~m}$ to 1.5 $\mathrm{m}$ (Fig. S2d-f). Increasing the width of leads decreases the size of the open water region (Fig. S2f), leading to a slower increase in ice concentration. After 15 days, ice concentration decreases by $43 \%$ as $r_{\mathrm{lw}}$ increases by $200 \%$ (Fig. S2e).
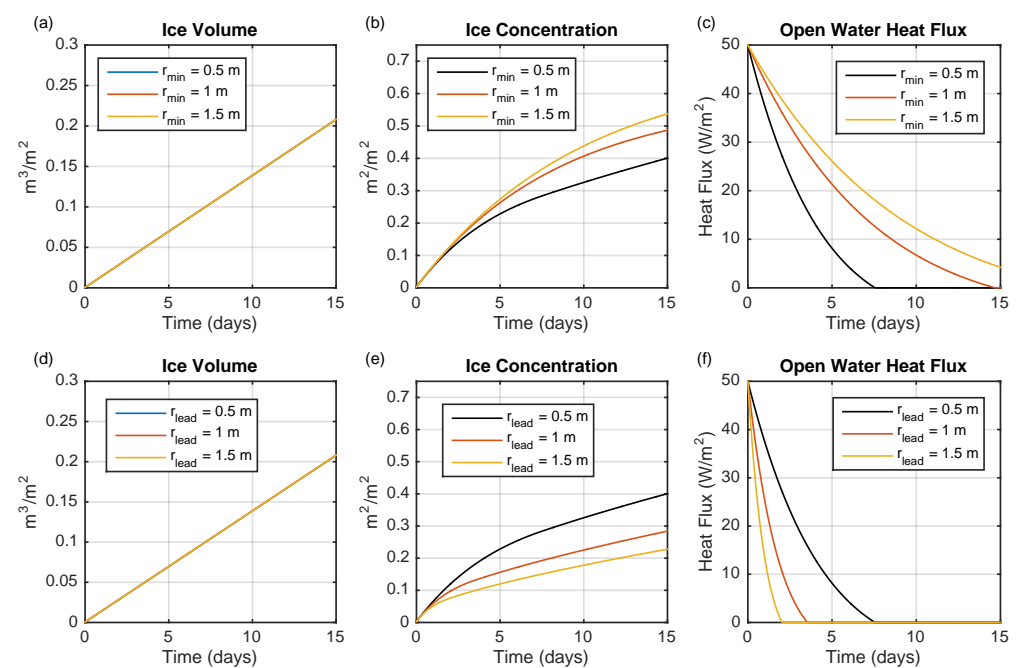

Figure S2: Sensitivity to the variation of the lead width $r_{\mathrm{lw}}$ and minimum floe size $r_{\min }$, for an initially uncovered sea surface with a net applied cooling of $50 \mathrm{~W} / \mathrm{m}^{2}$. (a) Ice volume, (b) ice concentration, and (c) open water heat flux, for runs in which $r_{\min }$ is varied from $0.5 \mathrm{~m}$ to $1.5 \mathrm{~m}$. (d-f) Same as (a-c), but when $r_{l w}$ is varied from $0.5 \mathrm{~m}$ to $1.5 \mathrm{~m}$. Black lines correspond to default model values, $r_{l w}=r_{\min }=0.5 \mathrm{~m}$.

\section{S1.2 Mechanics}

This set of runs is initialized as in Sec. 3 of the manuscript, with two Gaussian peaks in the FSTD. The first peak has a mean size of $90 \mathrm{~m}$ and a mean 
thickness of $0.25 \mathrm{~m}$. The second peak has a mean size of $15 \mathrm{~m}$ and a mean thickness of $1.5 \mathrm{~m}$, and only the mechanical component of the model is enabled. The external forcing is defined to be a set convergence of $1 \times 10^{7}$ $1 / \mathrm{s}$, applied for 30 days. These model runs are performed using the original floe size discretization outlined in the manuscript, spaced according to

$r_{n+1}=\sqrt{6 / 5} r_{n}, r_{1}=0.5 \mathrm{~m}$, with 64 bins up to $156 \mathrm{~m}$. Each run has an evenly spaced grid of 14 floe thicknesses from $0.1 \mathrm{~m}$ to $2.7 \mathrm{~m}$.

Parameters that influence the mechanical component of the FSTD model are the widths $\left(\delta_{\text {ridge/raft }}\right)$ of ridges and rafts formed in floe collisions, and the thickness multiple $k_{\text {ridge }}$, the ratio of the thickness of a new ridge to the thickness of the smallest of two combining floes. Since we represent rafting and ridging similarly in the model, we examine only sensitivity to ridging parameters. The ridge width is varied from 3 to 7 meters, and the ridging thickness multiple is varied from 3 to 7 . The response is seen in Fig. S3, note that the vertical scale is logarithmic.

The influence of changing the ridging multiple is minor, with little impact on either the FSD or ITD after 30 days (Fig. S3a,c). Changing the ridge width (Fig. S3b,d) influences the spread of smaller floes to larger sizes, and increasing the ridge width leads to more floes at smaller sizes, though the major differences are seen at sizes and thicknesses with concentration less than $1 \%$, so the model results are largely insensitive to these parameters.

\section{S1.3 Wave-induced fracture}

This set of runs is initialized with a single Gaussian peak in the floe size distribution at $90 \mathrm{~m}$ size and $1 \mathrm{~m}$ thickness. The fracture component of the FSTD model is turned on, and all other model components are turned off. The model discretization is the same as in Sec. S1.2. The external forcing consists of a Bretschneider surface wave spectrum, with a zero-crossing period of $6 \mathrm{~s}$ and a significant height of $2 \mathrm{~m}$, and is continuously applied for seven days at the ice edge. The model domain width is $10 \mathrm{~km}$.

Model parameters that influence the response of the FSTD to fracture of ice by ocean surface waves are the group velocity of waves in ice, $v_{\mathrm{g}}$ and the flexural strain failure threshold $\epsilon_{\text {crit }}$. The wave group velocity is varied from 0.5 to 1.5 times the surface gravity wave group velocity. The failure threshold is varied over an order of magnitude from $5 \times 10^{-6} 1 / \mathrm{s}$ to $5.5 \times 10^{-5} 1 / \mathrm{s}$. The response to the variation of these two parameters is shown in Fig. S4.

The group velocity changes the fraction of the model domain affected by 

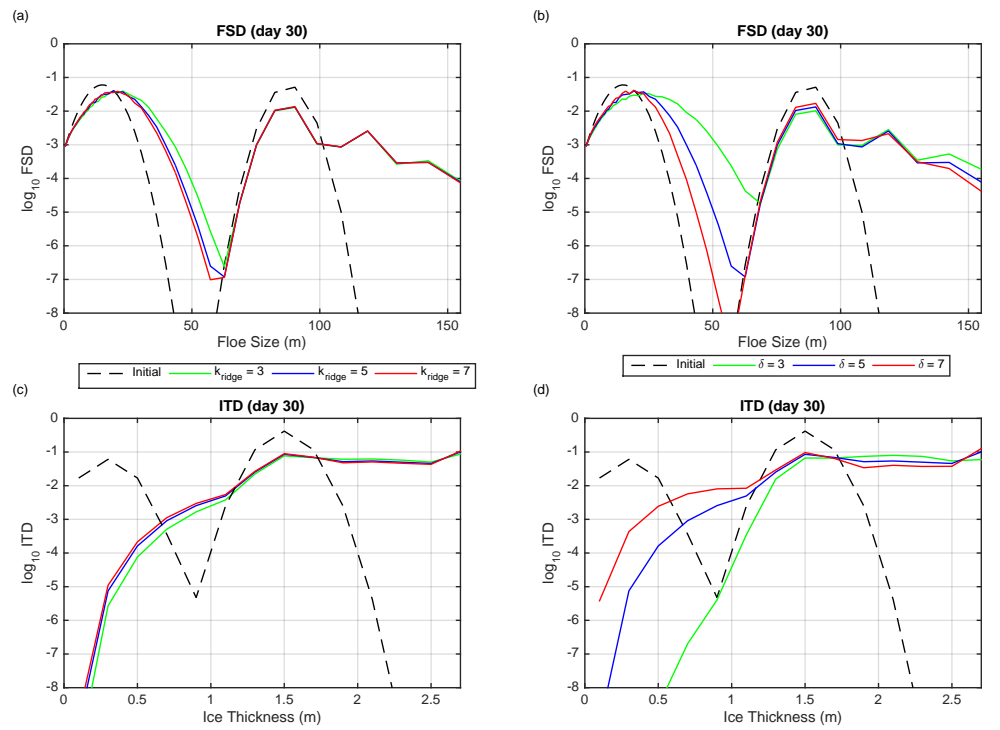

Figure S3: Sensitivity to the variation of ridge width and thickness multiple for the mechanical sensitivity run. (a,c) Base $10 \log$ of the FSD (a) and ITD (c) after 30 days, when the ridging thickness multiple is changed from 3 to 7. Dashed black line is the initial condition. (b,d) Base $10 \log$ of the FSD (b) and ITD (d) after 30 days, when the width of ridges is changed from 3 $\mathrm{m}$ to $7 \mathrm{~m}$ 

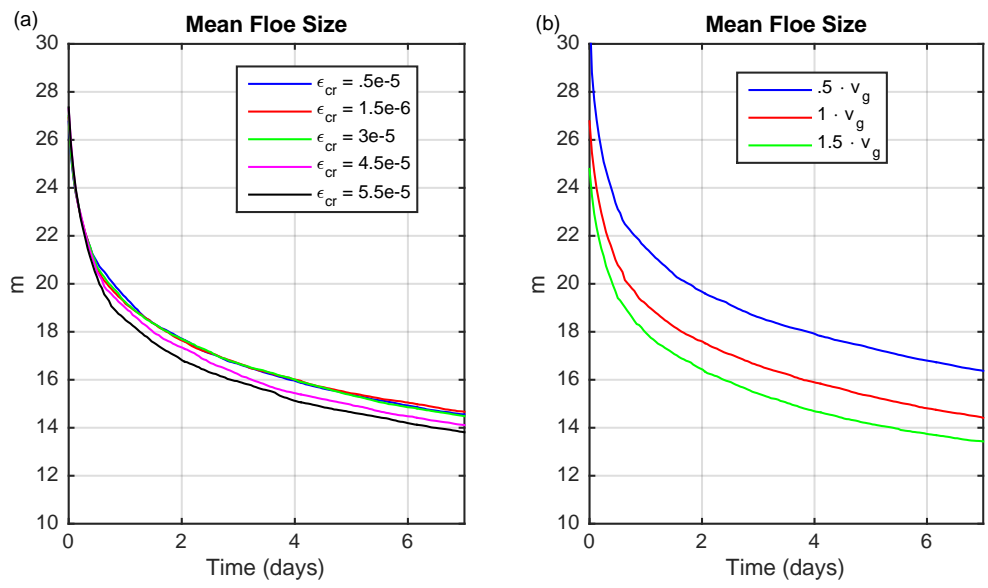

Figure S4: Sensitivity to the variation of parameters for runs with waveinduced fracture only, with a single Gaussian peak in the FSTD and 7 days of wave forcing. (a) Sensitivity of mean floe size to changes in $\epsilon_{\text {crit. }}$ (b) Sensitivity of mean floe size to the wave group velocity $v_{\mathrm{g}}$.

fracturing and therefore the time-scale of breaking. There is limited sensitivity to changes in the critical strain coefficient, as strains calculated are on the order of $10^{-2}$ and higher (Fig.'S4a), suggesting that further study of the critical strain necessary to break floes by waves is necessary. As the wave group velocity is increased, more fracture occurs and the mean floe size decreases (Fig. S4b).

\section{S1.4 Attenuation model}

Additionally, the parameterization of wave attenuation influences the response of the FSTD to the fracture of ice by ocean waves. We perform the same runs as in Sec. S2.2, comparing the wave attenuation model outlined in Bennetts and Squire (2012) (hereafter BS12) to the one-dimensional scattering attenuation model that is outlined and implemented in the main paper (Kohout and Meylan, 2008, herafter KM08). A comparison of the attenuation coefficient used as input in our FSTD model, as a function of wave period and ice thickness, is shown in Fig. S5 (compare with Fig. 1 in the main paper).

Fig. S6 shows how the mean floe size differs between the two simulations. The results from the run using BS12 (blue lines) have a mean floe size that 


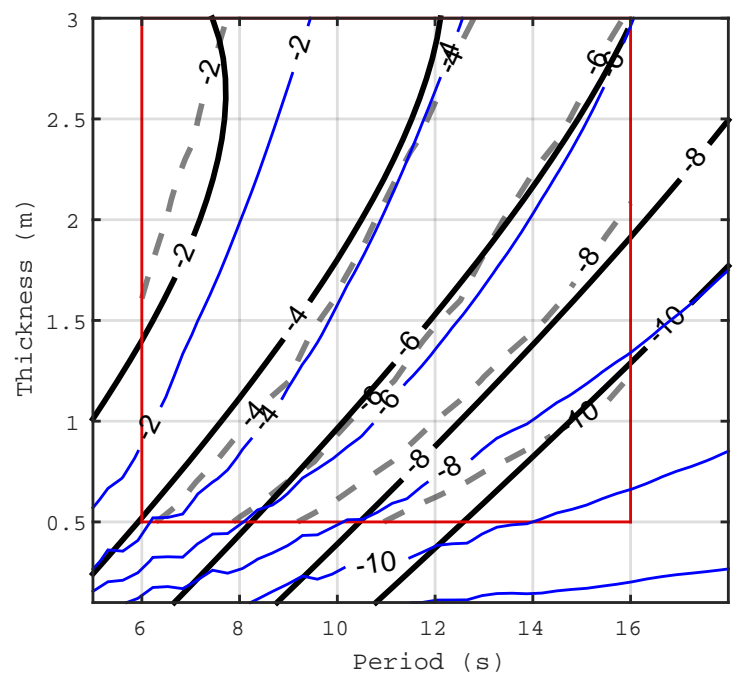

Figure S5: The natural logarithm of the attenuation coefficient $\alpha$ calculated by Kohout and Meylan (2008) (dash, inside the red box) and a quadratic fit to this attenuation coefficient that is used in section 4 of the manuscript (solid). Blue lines are the natural logarithm of the attenuation coefficient $\alpha$ as calculated by Bennetts and Squire (2012) and are not extrapolated. Solid contours outside of the red box are extrapolated using a quadratic fit. The fit is given by $\ln \alpha(T, \bar{h})=-0.3203+2.058 \bar{h}-0.9375 T-0.4269 \bar{h}^{2}+0.1566 \bar{h} T+$ $0.0006 T^{2}$.

is larger after one week than KM08 (red lines). The model results depend on the differences between the two parameterizations.

\section{S2 Numerical convergence tests}

To test for numerical convergence with respect to resolution in floe-size space, we examine two single-process runs (mechanics and wave-induced fracture), which are described below. The model runs are first performed using the original resolution used in the manuscript, spaced according to $r_{n+1}=\sqrt{6 / 5} r_{n}$, $r_{1}=0.5 \mathrm{~m}$, with 64 bins up to $156 \mathrm{~m}$. A second set of model runs is 


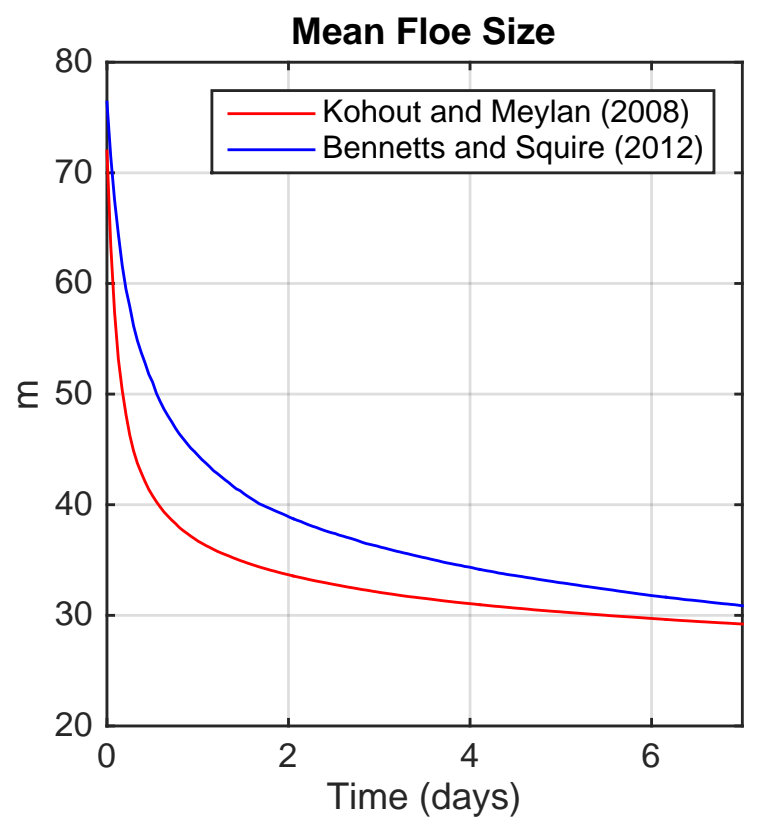

Figure S6: Comparison of the mean floe size in two week-long wave-induced fracture runs, using (red) the Kohout and Meylan (2008) or (blue) the Bennetts and Squire (2012) attenuation coefficient model. Both runs are initialized with a mean floe size of $87.5 \mathrm{~m}$, the first time plotted here is the first model time step, one hour after the initialization. 
performed using a doubled resolution, with 63 additional floe sizes spaced evenly between gridpoints of the original grid. These tests demonstrate the robustness of the model to changes in the grid resolution.

\section{S2.1 Mechanics run}

The initialization is as described in section S1.2. The mechanical interaction component of the model is turned on, while all other model components are turned off, with the results of this run shown in Fig. S7. The base 10
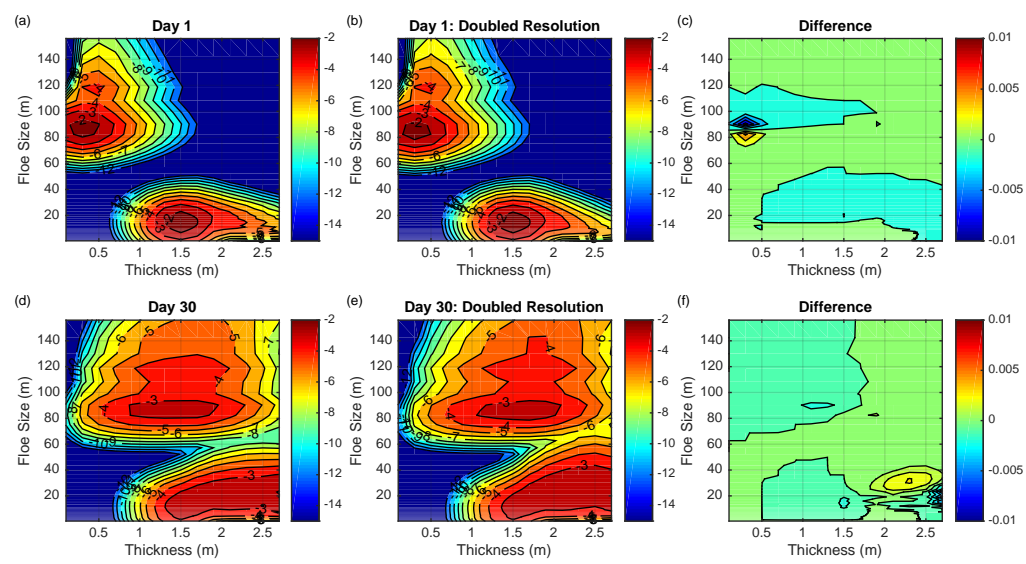

Figure S7: Base 10 logarithm of the FSTD for a regular resolution run $(\mathrm{a}, \mathrm{d})$ and doubled-resolution run (b,e) after 1 day (a-c) and 30 days (d-f). Contours are powers of ten in the distribution. (c,f) The difference (linear scale) between (a) and (b), and (d) and (e) when the doubled-resolution run is binned at the lower resolution.

logarithm of the FSTD after 1 day (Fig. S7a,d) and 30 days (Fig. S7b,e) are qualitatively similar. The difference between the two is calculated by binning the higher resolution into the lower resolution, and shows little difference between the two runs (Fig. S7c,f). The difference is nowhere larger than $1 \%$ in concentration after 30 days, so we conclude there is a limited sensitivity to resolution in these runs. 


\section{S2.2 Wave-induced fracture run}

The initialization used is as described in section S1.3, and the results are seen in Fig. S8. Fig. S8a shows the base 10 logarithm of the FSD over
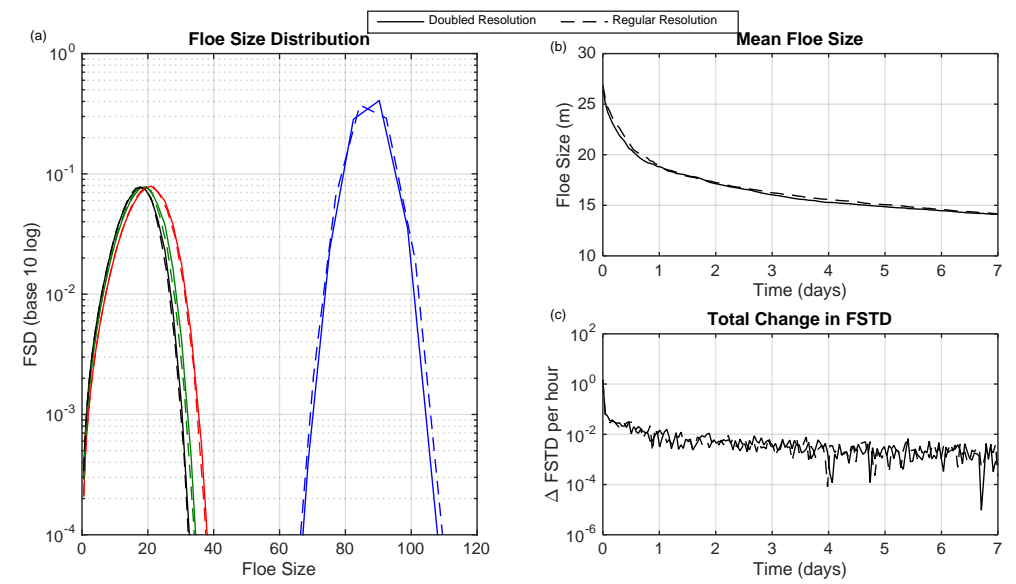

Figure S8: (a) Base 10 logarithm of the FSD for a regular resolution run (solid lines) and doubled-resolution run (dashed lines) at days 0 (blue), 2 (red), 4 (green) and 6 (black) of model runs that test convergence using wave-induced fracture alone. (b) The mean floe size over time for these runs. (c) The total fraction of the ice that is fractured, per day, for these runs.

time for the original (solid lines) and doubled (dashed lines) resolution runs, showing limited sensitivity to the shift in resolution. This is confirmed when examining the mean floe size (Fig. S8b), and additionally when examining the total area fractured per day (Fig. S8c), both of which are similar. We again conclude there is limited sensitivity to resolution in these runs.

\section{S3 Calculation of Fractured Floe Size Distri- bution}

We now demonstrate the wave fracture formulation with a specific example based on the Bretschneider wave spectrum $S_{B}(\lambda)$,

$$
S_{B}(\lambda)=\frac{H_{s}^{2}}{8 \pi} \frac{\lambda}{\lambda_{z}^{2}} e^{-\frac{1}{\pi}\left(\frac{\lambda}{\lambda_{z}}\right)^{2}},
$$




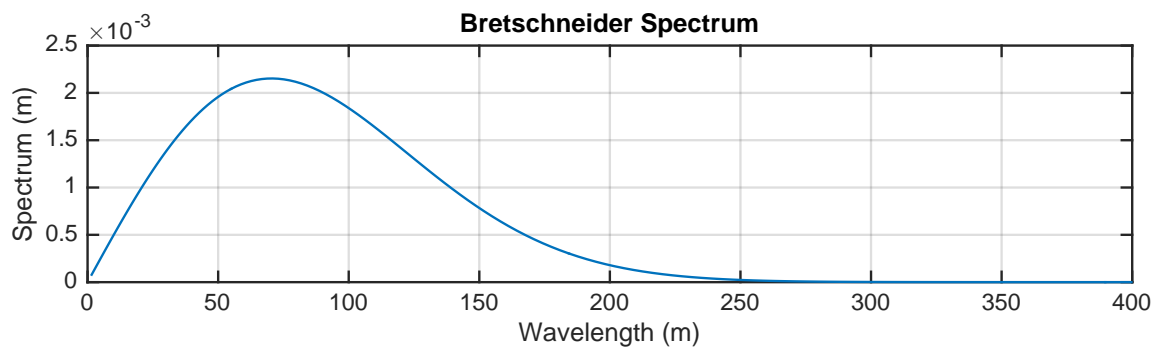

Figure S9: The Bretschneider wave spectrum $S_{B}(\lambda)$ (units of m) as a function of wavelength $\lambda$, for a significant wave height of $2 \mathrm{~m}$ and zero-crossing period of $6 \mathrm{~s}$.

shown in Figure S9, with units of $\mathrm{m}$, as a function of wavelength $\lambda$. The zerocrossing period $T_{z}$ is $6 \mathrm{~s}$ and significant wave height $H_{s}$ is $2 \mathrm{~m}$. The parameter $\lambda_{z}=g T_{z}^{2} / 2 \pi$ is the mean zero-crossing wavelength, $\lambda_{z} \approx 55 \mathrm{~m}$. Using minimum and maximum wavelengths of $\lambda_{\min }=.25 \mathrm{~m}, \lambda_{\max }=200 \mathrm{~m}$, and $\Delta \lambda=0.25 \mathrm{~m}$, spectral amplitudes are defined as $a_{i}=\sqrt{2 S_{B}\left(\lambda_{i}\right) \Delta \lambda}$, so that $\int S_{B}(\lambda) d \lambda \approx \sum_{i=1}^{N_{\lambda}} S_{B}(\lambda) \Delta \lambda=\sum_{i=1}^{N_{\lambda}} a_{i}^{2} / 2$. The domain size is $D=10 \mathrm{~km}$, and $x=0$ corresponds to the boundary from which waves enter the domain. There are $N_{\lambda}=\lambda_{\max } / \Delta \lambda=800$ spectral lines, and a realization of the sea surface height $\eta(x)$ is generated according to,

$$
\eta\left(x_{j}\right)=\sum_{i=1}^{N_{\lambda}} a_{i} e^{-\alpha\left(\lambda_{i}\right) x_{j}} \cos \left(\frac{2 \pi x_{j}}{\lambda_{i}}+\phi_{i}\right)
$$

where $\alpha\left(\lambda_{i}\right)$ is the attenuation coefficient for waves of wavelength $\lambda_{i}$, and the random phases $\phi_{i}$ are drawn from a uniform distribution between 0 and $2 \pi$. The first $100 \mathrm{~m}$ of a sample generated $\eta(t)$ is plotted as Fig. S10 (blue line). We assume that the floe conforms to the sea surface, and that it will fracture when it is strained between three successive local extrema of $\eta$ (Fig. S10, circles), where points are defined to be extrema when they are a local maximum or minimum over a distance of $10 \mathrm{~m}$ on each side. For a triplet of successive extrema (max, min, max; or min, max, min) of $\eta,\left(x_{i-1}^{*}, x_{i}^{*}, x_{i}^{*}\right)$, the strain felt by the floe at $x_{i}^{*}$ is calculated by the finite difference approximation,

$$
\epsilon\left(x_{i}^{*}\right)=\frac{h}{2} \frac{\partial^{2} \eta}{\partial x^{2}} \approx \frac{h}{2} \frac{\eta\left(x_{i-1}^{*}\right) \Delta x_{i+1}^{*}-\eta\left(x_{i}^{*}\right)\left(\Delta x_{i+1}^{*}+\Delta x_{i}^{*}\right)+\eta\left(x_{i}^{*}\right) \Delta x_{i}^{*}}{\Delta x_{i}^{*} \Delta x_{i+1}^{*}\left(\Delta x_{i}^{*}+\Delta x_{i+1}^{*}\right)}
$$




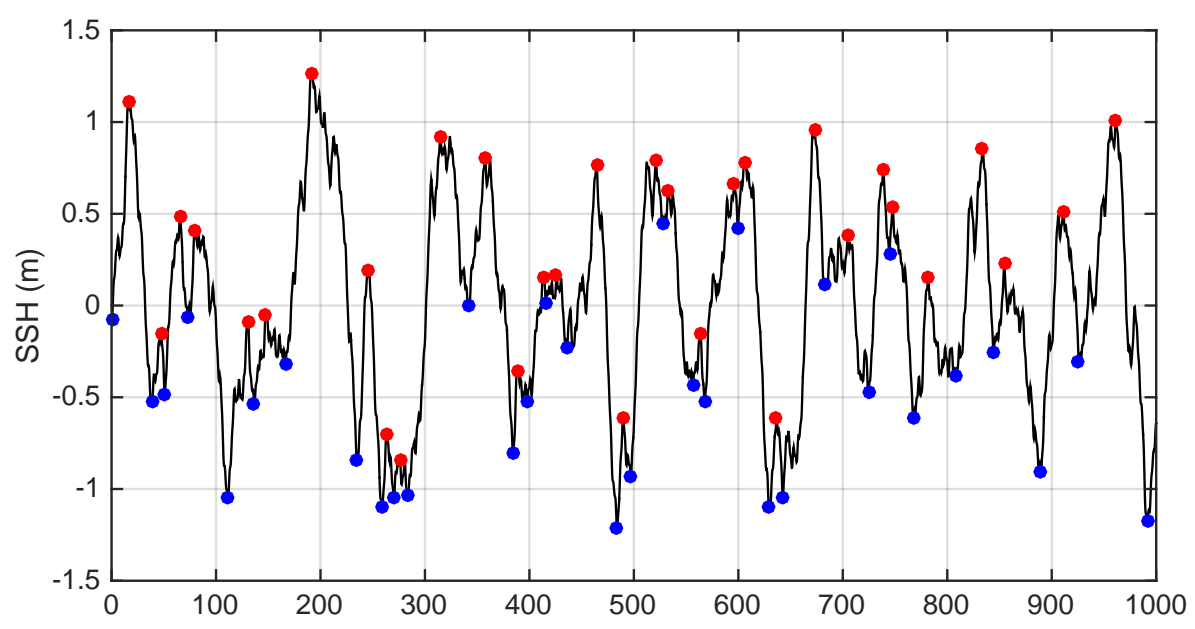

Figure S10: A sample sea surface height $\eta(x)$ calculated using the Bretschneider wave spectrum shown in Fig. S9. Shown is the first $1000 \mathrm{~m}$ of the wave record. Circles indicate local extrema which are used for calculating strain and subsequent floe sizes.

where $\Delta x_{i}^{*} \equiv x_{i}^{*}-x_{i-1}^{*}$. When the magnitude of this strain exceeds the critical strain, $\epsilon_{\text {crit }}=3 \times 10^{-5}$, the floe will break. This determines a set of points at which a floe of thickness $h$ will fracture, $X_{i}^{*}(h)$. From this set of points we define the size of the fractured floe as $X_{i+1}^{*}-X_{i}^{*}$. We form a histogram $R(r, h)$ of the number of occurrences of each fracture of size $r$, which is normalized so that $\int r R\left(r, h_{s}\right) d r=D$. In this way, $R\left(r, h_{s}\right) d r$ is equal to the number of fractures with size between $r$ and $r+d r$ and thickness $h_{s}$ when waves affect a fully ice-covered domain of length $D$. Figure S11 shows $R(1 \mathrm{~m}, r)$ for realizations of the sea surface using the Bretschneider spectrum seen in Fig. S9. The histogram is calculated on a uniform grid in $r$ of $1.3 \mathrm{~m}$ resolution and then smoothed and interpolated to the high resolution of the FSTD model.

We assume that a floe of size $s$ will fracture only when $X_{i+1}^{*}-X_{i}^{*}=r<s$, and that the number of fractures of size $r$ is either proportional to $R(r)$ (for $r<s$ ), or zero (for $r>=s$ ). The total length of fractures of size $r$ is thus proportional to $r R(r)$, or zero, for $r>s$. The floe size distribution formed by the fracture of a floe of size $s, F(\mathbf{s}, \mathbf{r})$ is therefore equal to the total length of floes of size $r$ that are formed by this fracturing of a floe of size $s$, normalized 


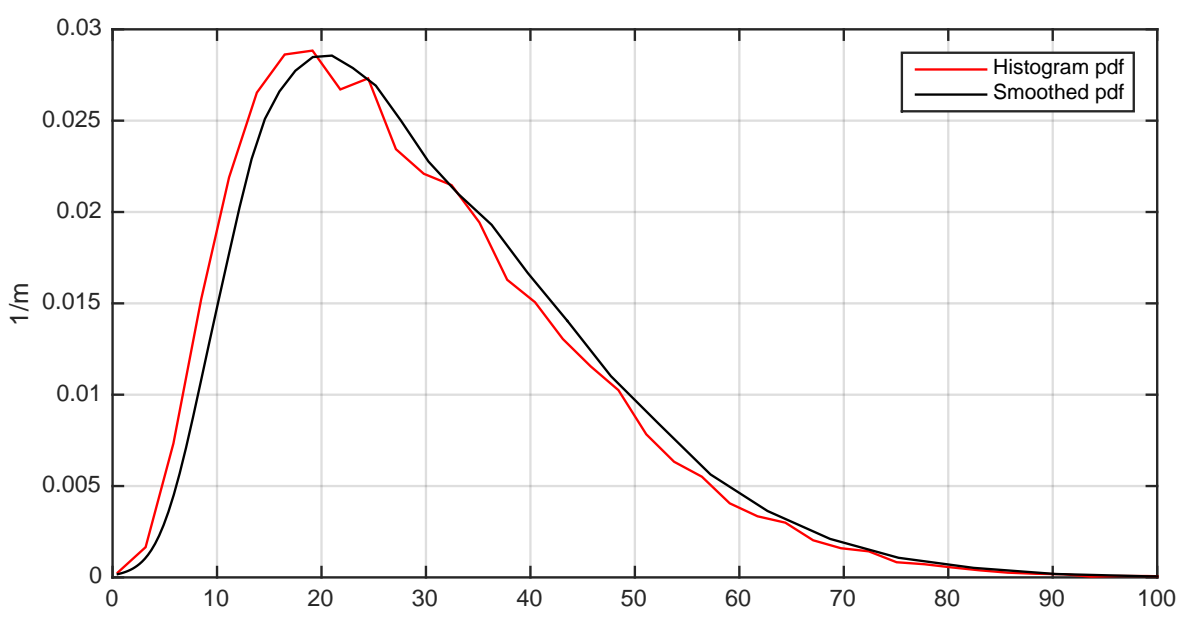

Figure S11: (red line) The probability (divided by the bin width) of a floe fracturing into each size class, calculated after 200 realizations of the sea surface height, with a uniform spatial discretization of $1.3 \mathrm{~m}$. (Black line) The same pdf smoothed and interpolated to the grid used in the FSTD model.

such that $\int_{0}^{\infty} F(\mathbf{s}, \mathbf{r}) d \mathbf{r}=1$, i.e.,

$$
F(\mathbf{s}, \mathbf{r})=F\left(\left[s, h_{s}\right],[r, h]\right)=\frac{r R\left(r, h_{s}\right)}{\int_{0}^{s} r R\left(r, h_{s}\right) d r} \delta\left(h-h_{s}\right) .
$$

The upper limit of the normalization integral in the denominator is truncated to $s$ because the integrand vanishes for larger values of $r$ as explained above. The delta function $\delta\left(h-h_{s}\right)$ represents the fact that fracture does not change ice thickness, i.e., any floes formed from the fracture of ice with thickness $h_{s}$ will also have thickness $h_{s}$.

The function $\Omega(\mathbf{r}, t) d \mathbf{r}$ is the fractional area that belongs to floes of size between $\mathbf{r}$ and $\mathbf{r}+d \mathbf{r}$ that is fractured per unit time. It is set equal to the the area fraction covered by floes of size $\mathbf{r}, \mathrm{f}(\mathbf{r})$, multiplied by the fraction of the domain reached by waves of group velocity $c_{g}$ per unit time, $c_{g} / D$, multiplied by the probability that floes of size $\mathbf{r}$ will fracture by waves. To calculate this probability, we note that $r^{\prime} R\left(r^{\prime}\right)$ is the total length of the domain covered by waves that can break floes into size $r^{\prime}$. Integrating this 
over $r^{\prime}$ from zero to a size $r$ we find the total width of the domain covered by waves that can produce floes smaller than $r$, which is the same as the length of the domain covered with waves that can break floes of size $r$ into smaller sizes. Normalizing by the domain width $D$, we find the final factor in the expression for $\Omega$,

$$
\Omega([r, h], t)=f(\mathbf{r})\left(c_{g} / D\right)\left(\int_{0}^{r} r^{\prime} R\left(r^{\prime}, h\right) d r^{\prime} / D\right) .
$$

The group velocity is taken to be that of the mean zero-crossing wavelength, $c_{g}=\sqrt{\frac{\lambda_{z} g}{8 \pi}}$. For the spectrum shown in Fig. S $9, c_{g} \approx 4.5 \mathrm{~m} / \mathrm{s}$. For the same spectrum, a sample calculated floe size distribution formed from the fracture of a floe of size $r=100 \mathrm{~m}$ and thickness $h=1 \mathrm{~m}, F([r, 1 \mathrm{~m}],[100 \mathrm{~m}, 1 \mathrm{~m}])$, is shown as Fig. S12. The third factor in Eq.4 is plotted as Fig. S12b for the same realizations.
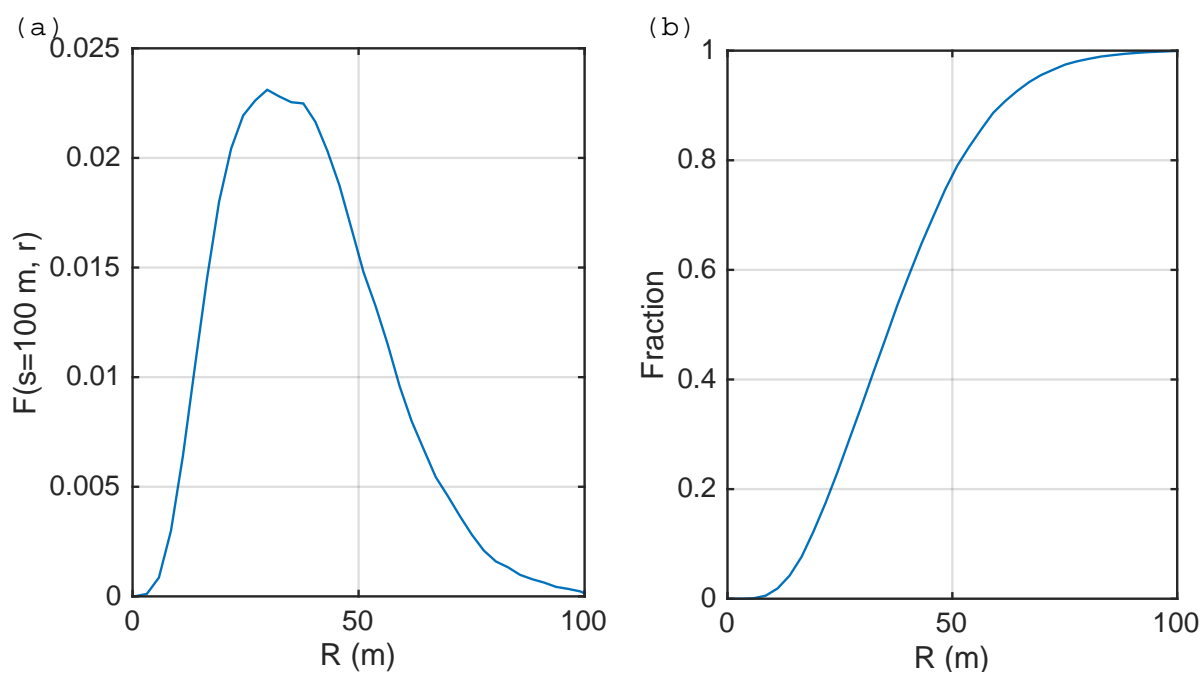

Figure S12: (a) The floe size distribution $F([100 \mathrm{~m}, 1 \mathrm{~m}],[r, 1 \mathrm{~m}])$ formed by fracturing a floe of size $100 \mathrm{~m}$ and thickness $1 \mathrm{~m}$ by the wave spectrum shown in Fig. S9. (b) The third factor in eq. 4, for the same wave spectrum and a domain width of $10 \mathrm{~km}$. 


\section{References}

Bennetts, L. G. and Squire, V. a. (2012). Model sensitivity analysis of scattering-induced attenuation of ice-coupled waves. Ocean Modelling, 4546:1-13.

Kohout, A. L. and Meylan, M. H. (2008). An elastic plate model for wave attenuation and ice floe breaking in the marginal ice zone. J. Geophys. Res., 113(9):C09016. 